\title{
Communication publique et responsabilité citoyenne
}

Ritha Cossette, Professeure (retraitée), Université du Québec à Montréal, rithacossette@hotmail.com 


\title{
Résumé.
}

Cet article examine le lien théoriquement indissoluble entre la communication publique et le paradigme éthique de la responsabilité : qu'est-ce qu'une communication publique responsable, ce à quoi s'engage implicitement tout professionnel de la communication ? Cette responsabilité se fait citoyenne dès lors que c'est la démocratie elle-même qui autorise l'exercice professionnel du métier de communiquer. Une structure à double renvoi ouvre à terme sur une autre éthique, celle plus fondamentale de la dette. Si en effet une société garantit les conditions de la communication publique, elle est en droit d'exiger en retour le respect de ses principes fondamentaux : outre l'État de droit, la pluralité légitime des points de vue, la véracité et la justesse du discours, le respect de l'intelligence des autres et, par extension, des différents publics auxquels elle s'adresse.

Mots-clés : communication, espace public, citoyenneté, éthique, responsabilité, dette.

\begin{abstract}
This article examines the theoretically indissoluble link between the public communication and the ethical paradigm of responsibility. What is responsible public communication, something which each communication professional implicitly commits to ? This responsibility applies to the citizen since it is democracy itself which authorizes the professional exercise of the task of communication. A structure with two way reciprocity leads to another fundamental consideration, that of the ethics of debt. If indeed a society guarantees the conditions of public communication, in return it requires respect for its fundamental principles: that of Rule of Law, legitimate plurality points of view, truthfulness and correctness of speech, respect for the intelligence of others and, by extension, for various publics that it addresses.

Mots-clés : communication, public communication, citizen, ethics, responsibility, debt.
\end{abstract}


« Autant peut faire le sot celui qui dit vrai que celui qui dit faux; car ce qui est en cause pour nous, c'est la manière de dire, non la matière ». Montaigne, L'art de la conversation.

«Vivons-nous actuellement dans une époque éclairée ?, on doit répondre : non, mais nous vivons dans une époque de propagation des lumières $»^{1}$. Cette question promptement répondue est déjà vieille de quelques siècles. Difficile de s'en débarrasser cependant dans la mesure où elle annonçait les conditions idéales d'une société authentiquement démocratique, ses valeurs fondamentales de liberté et de dignité absolue des personnes. La réponse est provocante mais sans doute vaudraitelle encore pour les citoyens d'aujourd'hui. Avons-nous en effet levé les obstacles majeurs à l'exercice de la démocratie et aménagé, pour nous-mêmes, cet espace de franche et libre discussion - un espace public - que Kant appelait de tous ses vœux ?

L'espace public, l'arène dans laquelle les citoyens débattent de façon la plus rationnelle possible de ce qu'ils ont en commun, est la condition première à l'exercice de la citoyenneté. Lieu de débats contradictoires pour comprendre ce qui arrive ou décider de ce qui devrait arriver; débats sur les conséquences graves et inquiétantes d'un projet ou d'une action collective ; débats sur la pertinence des lois et des autorités instituées, etc. D'où le caractère normatif de la discussion démocratique telle qu'elle est menée dans la société civile, dans les institutions publiques et dans l'État. La liberté d'expression et la circulation publique des idées - le principe de publicité -, la qualité de l'information, sa pertinence et sa justesse s'imposent alors dans les mentalités comme condition essentielle pour une saine gestion des organisations ou une gouvernance éclairée des sociétés (Dewey, 2005). D'où le droit et le devoir de s'informer - oser savoir, dirait Kant - et d'informer correctement.

Mais le monde a bien changé depuis Kant. Et la sphère publique est désormais investie par de multiples entrepreneurs et réseaux d'information, journalistes et citoyens-journalistes, chroniqueurs, commentateurs et analystes improvisés, politiques et intellectuels engagés, blogueurs et promoteurs aguerris d'opinions, relationnistes, publicistes et habiles faiseurs d'images. Autant d'agents qui se disputent âprement l'attention et l'adhésion du public à une idéologie, à une cause ou à une autre. Et c'est sans compter le recours massif des citoyens aux médias sociaux. Mais alors, comment se fier à ce qui se dit dans cet espace bruyant et encombré ?

Le présent article examine le lien théoriquement indissoluble entre la communication publique et le paradigme éthique de la responsabilité. La communication publique généralisée (l'exercice public de la raison dirait Kant) réfère pour nous aujourd'hui à ces différentes pratiques enchevêtrées et dont la communication professionnelle des institutions et des administrations serait l'une de ses composantes catégorielles. Qu'est-ce qu'une communication publique responsable, ce à quoi s'engage implicitement tout professionnel de la

\footnotetext{
${ }^{1}$ Kant, E. Réponse à la question : Qu'est-ce que les lumières ?, Bibliothèque de la Pléiade, Ed. Gallimard, 1985, p. 215.
} 
communication? Sur quoi reposent la responsabilité et l'imputabilité de qui a choisi le difficile métier de communiquer?

La régulation déontologique des pratiques de communication dispose non seulement à une plus grande probité intellectuelle mais invite aussi à une honnête contribution à la vie démocratique. L'élaboration déjà avancée de chartes ou de textes normatifs promet alors d'affermir sinon de rescaper une identité et un statut professionnels précaires. Le domaine des relations publiques qui nous occupera ici ne fait évidemment pas exception, même si cela vaut pour tous les métiers de l'information qui, il faut bien l'admettre, participent dorénavant d'un redoutable mélange des genres. Il est tout de même possible de distinguer certaines postures de communication.

\section{Postures de communication dans l'espace public}

La posture de communication la plus objective possible et aussi la plus dubitative, renvoie à l'activité journalistique : enquêtes rigoureuses et approfondies sur des questions d'intérêt général, reportages ponctuels sur des évènements, des faits problématiques, des dysfonctionnements démocratiques, etc. Cette posture et la déontologie correspondante relève d'un idéal d'exactitude et d'impartialité et trouve sa légitimité dans le droit du public à l'information ${ }^{2}$. Il en va de même pour le domaine plus restreint des communications institutionnelles. Formelles, méthodiques et informatives, celles-ci concernent le fonctionnement interne de l'État, ses missions, ses orientations et ses programmes ou celui de différentes instances publiques. Comme pour l'information journalistique, la communication institutionnelle à destination des citoyens est liée au principe de neutralité et doit d'abord servir l'intérêt général des populations desservies (Zemor, 1996).

Les relations publiques présentent à cet égard un double profil, œuvrant, soit en fonction de l'intérêt général pour autant qu'une autorité ou une institution publique soit concernée, soit selon des intérêts particuliers, multiples et contradictoires au cœur même de la société civile : entreprises, fondations, réseaux communautaires, associations, syndicats, groupes sociaux, etc. Bien que les relations publiques intègrent et respectent une certaine factualité, elles sont d'abord menées selon une logique de l'intérêt et en fonction de publics circonscrits. Adoptant la posture de communication pour convaincre ou pour promouvoir ces différents intérêts, elles peuvent compter ou miser sur les ressources infinies de la sophistique ou de la rhétorique. Elles dépassent en cela largement la seule visée informative.

Aussi, les opérations de relations publiques sont-elles maintenant nécessaires pour ménager à une organisation une place concurrentielle dans le marché des services, des

\footnotetext{
${ }^{2}$ Pour un examen approfondi de la déontologie professionnelle des journalistes, consulter l'ouvrage de Daniel Cornu, Journalisme et vérité, L'éthique de l'information au défi du changement médiatique, Labor et Fides, 2009.
} 
biens et des idées, pour promouvoir une marque, un programme, une personnalité ou un parti politique. Opérations délicates pour prévenir ou contenir une crise, résoudre un scandale, atténuer la violence médiatique ou la vindicte populaire, redresser une réputation malmenée, etc. Parfois consensuelles, elles se réalisent en fonction de ce qui préoccupe un groupe ou une population. Relations publiques pour rassurer et inspirer confiance. Parfois acrimonieuses et réactives, elles relayent et importent dans l'espace public la colère ou l'indignation d'un groupe à destination d'un autre ou d'une autorité. Relations publiques pour apaiser une opinion populaire récalcitrante ou pour redéfinir un rapport de force. Aucune organisation moderne ne pourrait manifestement se développer sans ce processus de communication soutenue avec différents publics, communautés, réseaux ou collaborateurs.

Combien de fois n'a-t-on pas assimilé cependant cette action toujours déjà intéressée, à une propagande mensongère ou à la subtile manipulation des esprits et de l'opinion publique ? La cause est entendue. C'est ce déficit de crédibilité qui a forcé l'élaboration de standards déontologiques davantage respectueux des droits ou de la protection du public; plus respectueux aussi des différents systèmes de collaboration et de standards professionnels communs.

Cette responsabilité d'une parole fiable, juste et crédible d'une organisation incombe pour une bonne part au relationniste qui, à la faveur d'une formation universitaire plus affirmée, endosse selon les aléas des circonstances et des mandats, la figure fuyante du stratège, de l'analyste ou de l'expert-conseil. Où commence et où finit cette responsabilité ? Que signifie plus fondamentalement être responsable?

\section{Le paradigme éthique de la responsabilité}

On retrouve, selon les époques et les disciplines universitaires, différentes approches de l'idée de responsabilité. Il est tout de même possible, au delà d'un flou conceptuel persistant entourant cette notion, d'en dégager les lignes générales.

Le principe de responsabilité, condition nécessaire bien qu'insuffisante à l'organisation de la vie sociale, repose sur une double normativité. La forme qui nous est la plus familière est sans doute la responsabilité juridique enchâssée dans les lois, elles-mêmes consignées dans des codes civils ou criminels. Cette forme de responsabilité est pour l'essentiel limitée aux effets nuisibles des actions et à la sanction des fautes : « En un sens strictement juridique, l'imputation présuppose un ensemble d'obligations délimitées négativement par l'énumération précise des infractions à la loi écrite, à quoi correspond l'obligation en droit civil de réparer le tort commis et en droit pénal celle de se soumettre à la peine. Est réputé imputable le sujet placé sous l'obligation de réparer les dommages et de subir la peine $»^{3}$. Il faut cependant distinguer les deux ordres différents de l'imputabilité et de la

${ }^{3}$ Ricoeur, P. Parcours de reconnaissance, Gallimard, 2004, p. 172. 
responsabilité: celle-ci renvoie au sujet tandis que l'imputabilité concerne un système de jugement qui lui est extérieur (Genard, 2006). L'imputabilité réfère principalement, par ailleurs, aux actions passées alors que la responsabilité est davantage contemporaine de l'action.

La deuxième modalité, la responsabilité morale, est à plusieurs égards illimitée dans la mesure où elle engage l'ensemble de nos rapports à autrui, notre rapport au monde. $\mathrm{Ce}$ deuxième versant de la responsabilité recouvre la deuxième distinction problématique entre responsabilité morale individuelle et collective. Qu'en est-il dans un premier temps de la responsabilité à la première personne ?

L'éthique de la responsabilité implique, avant de l'appliquer à quelque domaine professionnel que ce soit, une première référence directe à l'agent : qui est celui qui agit et qui peut-être tenu responsable de ce qui arrive ? L'idée de responsabilité n'a donc de sens que dans la relation éthique à l'autre. Où commence l'éthique et pourquoi faut-il y revenir sans cesse?

L'éthique s'installe d'abord dans les esprits bien davantage que dans les codes ou dans des textes normatifs. Kant fixe son début décisif par la sortie hors d'un état de minorité - l'abandon d'une posture passive et infantile -, par le refus de se voir et de se présenter comme simple exécutant assujetti à la volonté d'un autre. Se conformer à des rôles sociaux ou professionnels prédéterminés ne sera jamais suffisant. Et l'autonomie morale en cause ici repose sur la capacité fondamentale de se désigner comme un JE, une instance psychique qui pense, qui juge, qui parle en son nom. Etre responsable, c'est être un sujet : un Je particulier qui agit et qui assume le devoir de répondre de ses actes, qui donne la raison de ses choix (Genard, 2006).

Aussi, la responsabilité implique-t-elle une certaine posture, un certain maintien de soi révélateur de l'identité éthique telle que théorisée par Ricoeur: se tenir moralement responsable c'est « accepter d'être tenu pour le même aujourd'hui que celui qui a agi hier et qui agira demain, ... c'est pour la personne la manière telle de se comporter qu'autrui peut compter sur elle. Parce que quelqu'un compte sur moi, je suis comptable de mes actions devant un autre. Le terme responsabilité réunit les deux significations : compter sur ..., être comptable de ... $»^{4}$. Aussi bien dire d'ailleurs que ce maintien de soi est toujours contemporain de la tenue de ses engagements et de ses promesses : je ferai demain ce que je dis aujourd'hui que je ferai. Je m'y tiendrai.

L'agir responsable se révèle aussi, par delà ce laborieux procès de subjectivation le devenir adulte -, par la mobilisation de toutes ses capacités. L'idée n'est pas neuve. On la trouve notamment dans l'Art de la conversation de Montaigne selon qui « il faut qu'il y ait plus de vigueur et de puissance dans le porteur que dans la charge. Celui qui n'a pas mis en œuvre toute sa force, vous laisse deviner s'il en a encore au-delà et s'il a été mis à l'épreuve jusqu'à sa dernière possibilité » ${ }^{5}$. Et si nous sommes souvent irrités par la déresponsabilisation d'autrui, nous anticipons toujours, pour nous-

\footnotetext{
${ }^{4}$ Ricoeur, P., Soi-même comme un autre, Gallimard, 1990, p. 342.

${ }^{5}$ Montaigne, M. Les Essais, L'art de la conversation, Gallimard, 2009, p. 1128.
} 
mêmes, le fardeau qui accompagne le fait de devoir prendre ou de devoir assumer ses responsabilités.

Pour exemple, la décision hautement risquée du capitaine Sullenburger d'amerrir sur la rivière Hudson en 2009 plutôt que d'atterrir sur une piste pourtant disponible dans un aéroport à proximité. Un moment d'autant plus tragique que la vie de cent cinquante personnes étaient en danger. Lourde responsabilité que celle-là et aucune possibilité de se décharger sur quiconque, son employeur, ses collègues ou collaborateurs. Ce fardeau imposé par une situation grave et inédite et à laquelle il devait réagir, c'était à lui et à lui seul de le porter. À lui et à lui seul revenait la décision finale. Une décision qui, bien au-delà des lois, des normes et des protocoles, s'attachera à la personne du pilote et à ce qu'il a fait ce jour-là. Même impliqué dans une entreprise collective, même supporté par son syndicat ou son employeur, c'est lui-même - sa compétence et la qualité de son jugement - qui seront examinés, blâmés et éventuellement sanctionnés. On connaît la suite : il a dû s'expliquer devant le prestigieux NTSB quelques mois plus tard, organisme à son tour responsable de la sécurité aérienne. Il y allait de la réputation de sa compagnie voire de l'ensemble de l'industrie. De la relation de confiance surtout entre voyageurs et transporteurs, confiance plus immédiate dans la compétence professionnelle et le sens du devoir de leurs pilotes. Ce poids moral de la charge de l'autre, dans ce cas-ci un groupe de voyageurs confiants, constitue l'objet ultime de la responsabilité (Ricoeur, 2004).

Et la reconnaissance par l'agent lui-même d'être l'auteur de tel ou tel acte atteste implicitement qu'il en était capable : je l'ai fait, c'est donc que j'en étais capable ; c'est moi l'instigateur de ce qui est arrivé, c'est donc à moi que revient l'obligation d'en répondre. C'est cette reconnaissance- là qui dispose précisément à l'imputabilité.

Cette figure majeure de la responsabilité à la première personne serait toutefois bien insuffisante si nous en restions là. Le sujet n'a pas les ressources intérieures suffisantes pour assumer ou s'acquitter de tout. Ses compétences personnelles doivent en effet pouvoir se combiner avec des ressources extérieures disponibles dans l'environnement matériel, social et politique (Nussbaum, 2012). Nos environnements sont cependant devenus remarquablement complexes : multiplicité des intervenants s'enchevêtrant et s'entrechoquant même parfois les uns les autres; hiérarchie organisationnelle et organigrammes sophistiqués; coordination laborieuse des rôles et division aléatoire du travail; méthodes plus ou moins formelles et protocoles contraignants, etc.

Il faut donc lier l'assomption de la responsabilité à l'expérience associative ellemême - l'intersubjectivité - et qui va bien au-delà d'un simple partage des rôles et des prérogatives. Le postulat de l'intersubjectivité coiffe donc l'idée d'une subjectivité enclose sur elle-même héritée de la première modernité cartésienne. La signification, en d'autres mots, de ce qui est dit ou fait par les uns ou par les autres et qui exigent d'en répondre ne tient pas à un processus monologique : "Jamais solipsiste, toute action, aussi singulière et personnelle soit-elle, se déploie dans, et 
déploie avec elle, un champ qui implique une pluralité d'acteurs comme un pluralité de destinataires $»^{6}$.

Chose étonnante par ailleurs, le fait que les gens résistent la plupart du temps aux tentations de se décharger du fardeau de la responsabilité. Peut-être faut-il regarder en direction de la dynamique

interne des groupes sociaux qu'anime une incessante quête pour la reconnaissance. Il y va du développement et du maintien de l'estime de soi, de la possibilité de se reconnaître soi-même comme un être responsable : «De fait, la plupart des conflits qui surgissent typiquement au sein d'un groupe se situent sur un plan proche de la conscience et se laissent aisément reconstruire en termes rationnels. Ils sont en général liés à l'interprétation et à la hiérarchisation des prestations dont les sujets doivent pouvoir s'acquitter pour être considérés comme des membres reconnus du groupe ${ }^{7}$. Le souci et le sens de la responsabilité seraient assumés sinon assurés à la faveur précisément de l'expérience de l'appartenance à un groupe.

Comment circonscrire alors la part d'action de chacun? Peut-on d'un point de vue moral rendre quelqu'un responsable pour quelque chose qu'il n'a pas lui-même accompli ? Comment une communauté pourrait-elle ne pas se sentir responsable de l'action de l'un de ses membres ou de ce qui a été fait en son nom ? Question problématique et irrésolue que celle de la responsabilité collective et qui déborde largement notre propos.

Il est tout de même possible de voir la convergence entre les deux principes de la responsabilité et celui d'une nécessaire solidarité humaine : «Cette responsabilité déléguée pour des choses que nous n'avons pas faites, à savoir que nous prenions sur nous les conséquences de choses dont nous sommes entièrement innocents, est le prix que nous payons pour conduire notre vie non de façon indépendante, mais parmi nos congénères $»^{8}$. Cette proposition d'Arendt fait signe vers l'idée d'un monde commun et de la figure du citoyen qui doit s'en rendre responsable.

\section{La responsabilité citoyenne}

La responsabilité citoyenne atteste d'une loyauté première à des principes démocratiques en excès sur les intérêts corporatistes, particuliers et égocentrés de la communication publique. Et sans doute peut-on la référer à une responsabilité pour le monde. Une responsabilité que l'on pourrait dire globale (Gros, 2012). Un souci éthique fondamental pour ce qui se passe concrètement dans le monde, les choix périlleux pour l'avenir de l'humanité et pour la planète entière.

\footnotetext{
${ }^{6}$ Etienne Tassin, préface à Assumer l'humanité, Philosophie et société, p. 11.

${ }^{7}$ Honneth, A. Ce que social veut dire, Gallimard, 2015, p. 247/248.

${ }^{8}$ Arendt, H., Responsabilité et jugement, Éditions Payot \& Rivages, 2005, p. 183.
} 
Pas de démocratie véritable sans la montée en puissance de la parole citoyenne. Impossible alors de communiquer sans l'idée régulatrice de vérité : "Pour ne pas dire la vérité, il faut qu'il y ait une vérité à ne pas dire $»{ }^{9}$. L'idée de vérité même soumise à la dure critique des sceptiques semble toujours déjà vitale pour un juste rapport avec les autres et une conception adéquate du monde réel. Pour vivre ensemble. La responsabilité citoyenne prend alors les traits d'une responsabilité épistémique : le devoir de chercher et de s'efforcer de délivrer des informations qui soient justes, exactes et fiables : «Un individu qui, dans un contexte de confiance, agit consciencieusement avec le but d'apporter de l'information à autrui se donnera du mal pour s'assurer, dans une mesure raisonnable, que l'opinion qu'il transmet est vraie ; cela revient à dire qu'un investissement d'investigation peut se faire au bénéfice d'autrui ou au bénéfice du groupe $»^{10}$. La probité intellectuelle est à ce prix.

Aussi la théorie habermassienne de la communication insiste-t-elle sur l'intelligibilité et la justesse des énoncés : «Ceux qui participent à une interaction doivent se considérer réciproquement comme responsables et donc supposer qu'ils fondent leurs actes sur des prétentions à la validité »(Habermas, 1997). Parler de manière rationnelle et raisonnable suppose non seulement l'expression d'une pensée, mais, et surtout, l'identification à ce qui est dit : c'est bien moi qui dit et qui pense cela, qui pense à ce que je dis, là maintenant, et que je dis ce que je pense. Vraiment, en tout bonne foi.

Mais la probité et l'ouverture à la pluralité des points de vue ne vont pas de soi. Les stratégies ou les ruses langagières seront toujours possibles ou disponibles pour qui veut manipuler les esprits ou fuir ses responsabilités ${ }^{11}$. À commencer par le mensonge ou la dissimulation. Combien d'opérations de relations publiques ne sont-elles pas, à cet égard, blâmées pour leur caractère opportuniste et sournoisement intéressé, pour leurs pratiques antidémocratiques ? Combien de doutes sur l'authenticité des messages et des intentions?

Un principe fondateur de la vie en société commandera toujours pourtant de ne pas mentir, de ne pas déserter sa propre parole. Certains auteurs classiques dont Kant et Arendt ont lourdement insisté sur la gravité de cette désertion : « La puissance n'est actualisée que lorsque la parole et l'acte ne divorcent pas, lorsque les mots ne sont pas vides, ni les actes brutaux, lorsque les mots ne servent pas à voiler les intentions mais à révéler des réalités, lorsque les actes ne servent pas à violer et détruire mais à établir des relations et créer des réalités nouvelles ${ }^{12}$. Une sorte de contrat fondamental de

\footnotetext{
${ }^{9}$ Conche, M. Le fondement de la morale, PUF, 1993, p. 93.

${ }^{10}$ Williams, B., Vérité et véracité, 2006, p. 152.

${ }^{11}$ Les analyses de V. Jankélévitch dans L'ironie, Flammarion, 1964 et Les vertus de l'amour, Flammarion, 1986 sont particulièrement éclairantes sur ces différentes stratégies langagières. De même Perelman, C. et Olbretchs-Tyceta, Traité de l'argumentation, Éditions de l'Université de Bruxelles, 2008.

${ }^{12}$ Arendt, H., Condition de l'homme moderne. Calmann-Lévy, 1983, p. 260.
} 
confiance - confiance dans la parole de l'autre et dans ses promesses - précède ici tous les autres contrats possibles (Mangematin et Thuderoz, 2003).

Plusieurs raisons militent en faveur de la condamnation du mensonge : il ruine l'essence même de la parole censée exprimer une pensée et sur la base de laquelle s'établit la confiance mutuelle. Peut-on en effet se fier à quelqu'un qui dit le contraire de ce qu'il pense, coopérer avec lui ? Et paradoxalement, la personne qui ment ne souhaite pas du mensonge comme norme du discours refusant à autrui ce qu'elle s'autorise à elle-même ${ }^{13}$.

Et plus un réseau, une organisation, un groupe ou une personne a du pouvoir, plus grandes et parfois plus redoutables seront les conséquences du mensonge ou la dissimulation d'une vérité à laquelle les autres ont droit. Pour illustrer le propos, le cas hautement médiatisé de Lance Amstrong, cycliste vainqueur de sept tours de France $^{14}$. Une équipe de relationnistes et d'avocats ont dû manœuvrer pendant plusieurs années pour éviter que n'éclate au grand jour le scandale de dopage du célèbre et richissime athlète. La vérité a finit par triompher et Amstrong jusque là puissamment appuyé pas des commanditaires prestigieux a dû s'incliner et remettre ses médailles. De héros national qu'il était devenu il a été refoulé dans le camp peu enviable des menteurs et des tricheurs. Ses ambitions démesurées ont eu un effet dévastateur sur la vie et la carrière des coéquipiers qui ont refusé de participer à la manœuvre, de cautionner le plus grand et le plus nuisible des mensonges pour la discipline et sur l'ensemble des athlètes qui la pratiquent.

Le recours récurrent au mensonge repose sur des tendances psychosociologiques tenaces bien connues : il sert l'ego et son propre bonheur, apaise les peurs tenaces de l'échec, de la perte d'un amour ou de l'admiration des autres; mensonge altruiste aussi bien, dans l'intérêt ou par amour pour autrui : le pieux mensonge. Outre la considération qu'il est tout à fait possible de faire le bien d'autrui en lui mentant, il y a la croyance dans le fait que certaines personnes ou groupes de personnes n'ont pas droit à la vérité, qu'une vérité révélée pourrait toujours se retourner contre nous. Le débat philosophique autour de cette question oppose en fait le devoir de vérité au devoir de véracité : nous pouvons toujours nous tromper et nous nous trompons effectivement souvent, mais tromper délibérément autrui ou le public, est davantage sujet à désapprobation, moralement condamnable.

Mais, outre le mensonge dont on connaît les dangers quand il est dévoilé et toute l'énergie qu'il faut investir en relations publiques pour en réduire les effets nocifs, il existe un autre acte de parole commode, une autre stratégie de fuite de ses responsabilités : c'est le baratin, tout à l'opposé d'un raisonnement suivi en phase avec le monde réel. Combien de fois d'ailleurs les opérations de relations publiques n'ont-elles pas été discréditées pour leur caractère frivole et superficiel ? L'expression

\footnotetext{
${ }^{13}$ La critique kantienne du mensonge triplement contradictoire apparaît dans Sur un prétendu droit de mentir par humanité. La Pléiade tome III, 1986.

${ }^{14}$ Voir Holmes, Alex, Lance Amstrong : la victoire à tout prix, 2014.
} 
«Ce n'est qu'une opération de relations publiques » est pour exemple fréquemment utilisée entre adversaires politiques s'accusant mutuellement de berner la population par la multiplication de phrases creuses ou de propos insignifiants.

Baratiner, cela revient à parler mais ne rien dire ou à parler pour ne rien dire, s'exprimer de façon à ce que l'interlocuteur ne puisse exercer pleinement son intelligence critique. Celui ou celle qui baratine n'a d'ailleurs nul besoin d'introduire une fausseté dans le propos. Il ne veut ni dire ni cacher la réalité ou la vérité : il s'en moque. Le baratineur ne fait tout simplement pas attention à ce qu'il dit et sa marge de manœuvre est aussi grande que son habileté à puiser dans les ressources infinies du langage, à exploiter, mine de rien, la complaisance ou la crédulité de son public. Ennemie du baratin, une parole pour dire le vrai ou ce que nous croyons être vrai : la véridicité ; une parole articulée pour être compris, une parole intelligente pour être cru.

Ce sont l'ignorance et l'opinion irréfléchie qui expliquent, selon Frankfurt, l'omniprésence et le caractère inévitable du baratin qui apparaît « chaque fois que les circonstances amènent un individu à aborder un sujet qu'il ignore. La production d'inepties est donc stimulée quand les occasions de s'exprimer sur une question donnée l'emportent sur la connaissance de cette question. Ce genre d'écart est fréquent dans la vie publique, dont les acteurs sont portés - soit du fait d'un penchant naturel, soit en réponse à des demandes extérieures - à s'étendre sur des sujets malgré leur degré plus ou moins élevé d'ignorance $»^{15}$. Le discours bête ou insensé de celui qui « fait le sot» tient à une perversion de la parole, manœuvre d'autant plus facile et courante qu'il est de mise en démocratie d'avoir une opinion sur tout. D'où le sens qui peut être donné à la citation inaugurale de Montaigne : «Autant peut faire le sot celui qui dit vrai que celui qui dit faux ; car ce qui est en cause pour nous, c'est la manière de dire, non la matière ». Quelle manière? Quelle forme ?

La forme est le contraire de l'arrogance. Un citoyen éduqué, un esprit correctement formé prend la parole et sa parole au sérieux. Et Montaigne a depuis longtemps fixé la hauteur de l'enjeu : le respect de la parole et des promesses est la condition essentielle à l'édification d'un monde commun, du vivre ensemble : " Le commerce entre les hommes étant conduit par la seule voie de la parole, celui qui la fausse trahit la société publique. C'est le seul outil par le moyen duquel se communiquent nos volontés et nos pensées, c'est l'interprète de notre âme : s'il nous manque nous n'avons plus d'attaches entre nous, nous ne nous connaissons plus. S'il nous trompe, il rompt toute nos relations et délie tous les liens de notre société $»^{16}$. La franchise, la crédibilité et la confiance sont ici à prendre d'un seul tenant.

Un esprit correctement formé - une tête bien faite ! -, non seulement respecte sa parole mais aussi la parole des autres et leur état d'esprit, respect au fondement même

\footnotetext{
${ }^{15}$ Frankfurt, H. G., L'art de dire des conneries, Ed. 10/18, Département d'Univers Poche, 2006, p. 72.

${ }^{16}$ Montaigne, M., Les Essais, Sur le démenti, Gallimard, p. 810
} 
du lien démocratique : une parole en vaut une autre. Une personnalité démocratique, puisque c'est de cela dont il s'agit, s'ouvre aux questions dérangeantes, accepte dans l'argumentation de mettre en ballottage ses certitudes privées, ses préférences, ses prises de positions ou ses valeurs. Un esprit ouvert, non fanatique, non exagérément dogmatique, voit le monde en fait comme un laboratoire de recherche, là où rien n'est joué à l'avance, un monde opaque à enquêter sans jamais prétendre l'avoir cerner une foi pour toute. Il reconnaît, pour l'avoir lui-même expérimenté, le caractère transformateur du dialogue, la version la moins conflictuelle, la plus apaisée du rapport de communication (Gadamer, 2006).

La forme est aussi une question de jugement : juger c'est, non seulement se garder en phase avec le réel mais aussi, juger du point de vue des autres. Le recours au jugement et à l'éthique est d'ailleurs souvent motivé par l'effacement, la défaillance ou l'insuffisance des normes ou des déontologies, par le déphasage ou la distorsion entre les normes et la complexité sociale. Ils sont essentiels dans les moments de transitions ou de grands bouleversements susceptibles de compromettre le vivre ensemble et l'exercice de la citoyenneté. Il y a toujours besoin de juger correctement parce que les situations ne sont jamais si prévisibles et si nettes, parce que les choses ne se passent pas toujours comme nous l'avions anticipé ni dans les termes où nous l'aurions souhaité (Bergson, 1963). Jugement et responsabilité citoyenne sont foncièrement indissociables (Arendt, 1974). Comment asseoir dans l'opinion publique la crédibilité et la respectabilité d'un métier de communication, ici les relations publiques, sinon en s'acquittant de cette responsabilité citoyenne première.

Sur quoi repose in fine la responsabilité et l'imputabilité de qui a choisi le métier de communiquer? Peut-être cette question trouve-t-elle sa réponse dans une autre éthique que celle de la responsabilité mais qui ne lui est certainement pas étrangère : une éthique de la dette. La fameuse question «Qu'avons-nous que nous n'ayons point reçu? » de St-Augustin, que nous la dépouillions ou non de ses références religieuses initiales, jette les bases de cette éthique fondamentale. Aussi bien reconnaître que nous ne sommes jamais quittes de nos rencontres avec les autres, jamais si autosuffisants que nous puissions vivre humainement en dehors du système symbolique et culturel de la dette (Sarthou-Lajus, 1997).

Ainsi pourrions conclure en resserrant les termes de la discussion sur la responsabilité que ceux et celles qui font métier de communiquer ont précisément une dette envers la société démocratique qui en autorise l'exercice. L'éthique de la communication est l'éthique de la parole publique : la pervertir ou la dénaturer en manipulant par les mensonges, les inepties, l'insincérité, la duplicité, les duperies ou la mauvaise foi, revient à saper les fondements même de la démocratie, creuset de nos libertés fondamentales. Fragile, la démocratie qui est faite pour l'essentiel de relations ; incertaine aussi parce qu'elle peut toujours être instrumentalisée et détournée au profit d'intérêts dominants particuliers. Élever les pratiques de communication à la hauteur de ses standards, à savoir la condition de pluralité, le 
respect des libertés fondamentales, le droit à la vérité et la fiabilité de la parole, c'est s'en rendre activement responsable : une question d'intérêt bien compris.

\section{Bibliographie}

Arendt, H. (1989) Crise de la culture. Paris : Gallimard.

Arendt, H. (1974) Condition de l'homme moderne. Paris : Calmann-Lévy.

Chambers, S, « Rhétorique et espace public : La démocratie délibérative a-t-elle abandonné la démocratie de masse à son sort ? », in Raisons politiques. 2/2011 (n॰42), pp. 15-45.

Cornu, D. (2009) Journalisme et vérité. Le champ éthique, Genève : Labor et Fides.

Dewey, J. (2005) Le public et ses problèmes. Paris : Gallimard.

Foessel, M. (2008) La privation de l'intime. Paris : Seuil.

Génard, J.L., (1999) La grammaire de la responsabilité. Paris : Cerf.

Gros, F., (2012) Le sujet irremplaçable. Bibliothèque nationale de France.

Habermas, J. (2003) L'éthique de la discussion et la question de la vérité. Paris : Grasset.

Honneth, A. (2015) Ce que social veut dire, II. Les pathologies de la raison. Paris : Gallimard.

Jankélévitch, V. (1964) L'ironie. Paris : Flammarion.

Jankélévitch, V. (1986) Les vertus de l'amour. Paris : Flammarion.

Kant, E. (1985) Sur un prétendu droit de mentir par humanité. Paris : Gallimard, La Pléiade, tome III.

Mangematin, V., Thuderoz, C. (2003) Des mondes de confiance. CNRS Éditions.

Mead, G. H. (2006) L'esprit, le soi et la société, Paris : Presses Universitaires de France.

Montaigne, M. (2009) Les Essais, De l'art de conférer. Paris : Gallimard.

Nussbaum, C. M. (2012) Capabilités, Comment créer les conditions d'un monde plus juste? Paris : Flammarion, Climats.

Ricoeur, P. (2004) Parcours de reconnaissance, Paris : Gallimard, Éditions Stock.

Sarthou-Lajus, N. (1997) Éthique de la dette, Paris : Presses Universitaires de France. 
Communication publique et responsabilité citoyenne 27

Williams, B. (2006) Vérité et véracité, Essai de généalogie. Paris : Gallimard.

Zemor, P. Éthique et déontologie de la communication publique, LEGICOM 1/1996 $\left(\mathrm{N}^{\circ} 11\right)$, pp. 52-56. 